Pacific Journal of Mathematic 


\title{
STARSHAPED SETS AND THE HAUSDORFF METRIC
}

\author{
Gerald A. Beer
}

Let $C$ be a compact set in $R^{n}$. The $r$-parallel body of $C, B_{r}(C)$, is the union of the family of closed $r$-balls whose centers lie in $C$. If $C$ is starshaped with respect to the origin, the gauge of $B_{r}(C)$ is a Lipschitz function; this observation in conjunction with the Arzela-Ascoli theorem yields Blaschke selection theorem for starshaped sets. In addition, each parallel body is a union of a finite collection of parallel bodies of starshaped sets. From this decomposition, we show that Lebesgue measure is continuous on the metric space of parallel bodies of a fixed radius in $R^{n}$ relative to the Hausdorff metric.

1. Introduction. The dual cone of a convex set $C$ in $R^{n}$ is the epigraph of its support function. Using this concept Valentine [6] and others have obtained elementary proofs of many theorems in convexity.

The Blaschke selection theorem states that a uniformly bounded sequence of compact convex sets in $R^{n}$ contains a subsequence convergent in the Hausdorff metric to a compact convex set. The "compactness" of the corresponding sequence of dual cones follows immediately from the Arzela-Ascoli theorem: let $\left\{f_{n}\right\}$ be a pointwise bounded equicontinuous sequence of real-valued functions on a compact metric space. Then $\left\{f_{n}\right\}$ contains a uniformly convergent subsequence. The sequence of support functions corresponding to the particular convex sets under consideration clearly satisfy the hypotheses of the theorem. Extracting a convergent subsequence of functions, its limit must be a positively homogeneous subadditive function. Thus, the limit function is the support function of a compact convex set [6]. Since the Hausdorff distance between two compact convex sets is the supremum norm of the difference of their support functionals when restricted to the unit sphere, this set is easily seen to be the Hausdorff limit of the corresponding subsequence of convex sets. Of course, the Blaschke selection theorem remains valid when the assumption of convexity is deleted: each closed and bounded collection of compact sets in $R^{n}$ relative to the Hausdorff metric is compact [4]. Heil [3] in fact obtains this result by utilizing the Arzela-Ascoli theorem.

A main purpose of this article is to establish the selection theorem for starshaped sets by considering those functions intrinsically related to such sets: gauges. In light of the correspondence between gauges 
and support functions of polar convex sets, our method is in some sense a generalization of the one outlined above. Certain properties of parallel bodies of starshaped sets not only disclose the appropriate modifications required but also yield an interesting measure theoretic result for convergent sequences of parallel bodies of a fixed radius.

2. Preliminaries. Let $B_{r}(x)$ denote the closed $r$-ball about a point $x$ in $R^{n}$. If $C$ is compact set in $R^{n}$, then the $r$-parallel body of $C$, symbolized by $B_{r}(C)$, is the compact set $\mathrm{U}_{x \in C} B_{r}(x)$. If $C$ and $K$ are compact subsets of $R^{n}$, the Hausdorff distance of $C$ from $K$ is given by $d(C, K)=\inf \left\{r: B_{r}(C) \supset K\right.$ and $\left.B_{r}(K) \supset C\right\}$. If $\left\{C_{k}\right\}$ is a sequence of sets in $R^{n}$, then a point $x$ belongs to $\lim \sup \left\{C_{k}\right\}$ if and only if each neighborhood of $x$ intersects infinitely many terms of $\left\{C_{k}\right\}$.

Conv ker $C$ and conv $C$ will indicate the convex kernel and convex hull of $C$. Finally $x y$ denotes the line segment joining points $x$ and $y$, and $R(x, y)$ denotes the ray emanating from $x$ through $y$.

We now develop some properties of gauges of compact sets starshaped with respect to the origin.

Definition. Let $C \subset R^{n}$ be starshaped with respect to the origin. The gauge of $C$ is the function $g: R^{n} \rightarrow[0, \infty]$ defined by

$$
g(x)=\inf \{\lambda: x \in \lambda C\} .
$$

If $g$ is the gauge of a nontrivial closed set starshaped with respect to the origin, then $g$ is a nonnegative extended valued positively homogeneous lower-semicontinuous function, and there exists $x_{0} \neq 0$ satisfying $g\left(x_{0}\right) \neq \infty$. Conversely, any function $f$ with these properties is the gauge of such a set, namely, $C=\{x: f(x) \leqq 1\} . \quad C$ is closed as $f$ is lower-semicontinuous. Moreover, $0 \in C$ since

$$
f(0) \leqq \lim \inf _{k \rightarrow \infty} f\left(\frac{1}{k} x_{0}\right)=\lim _{k \rightarrow \infty} \frac{1}{k} f\left(x_{0}\right)=0 .
$$

The positive homogeneity of $f$ implies that $0 \in$ conv ker $C$. Note that $C$ is compact if and only if its gauge assumes the value zero only at the origin.

If $C$ is compact and $0 \in \operatorname{conv} \operatorname{ker} C \cap \operatorname{int} C$, then its gauge is finite valued. In this case a necessary and sufficient for continuity of the gauge is obtained from Dini's theorem: the gauges for $B_{1 / n}(C)$, $n=1,2,3, \cdots$, converge uniformly to the gauge of $C$ on compact subsets of $R^{n}$. If $0 \in \operatorname{int} \operatorname{conv} \operatorname{ker} C$, then the gauge is clearly continuous. Moreover, it is Lipschitz.

LEMma 1. Let $C \subset R^{n}$ be a compact starshaped set containing 
$B_{\varepsilon}(0)$ in its convex kernel. If $f$ is the gauge of $C$, then for all $z$ and $y$ in $R^{n}$

$$
|f(z)-f(y)| \leqq \frac{2}{\varepsilon}\|z-y\|
$$

Proof. For each $x \in C$, let $f_{x}$ denote the gauge of conv $\left(\{x\} \cup B_{\varepsilon}(0)\right)$. Clearly, $f_{x}(z) \geqq f(z)$ for all $x \in C$ and $z \in R^{n}$. For fixed nonzero $z$, choose $\lambda>0$ satisfying $\lambda z \in$ bd $C$. Then $f_{\lambda z}(z)=1 / \lambda=f(z)$ so that $f(z)=\inf _{x \in C} f_{x}(z)$.

We now show that the functions $\left\{f_{x}: x \in C\right\}$ are equi-Lipschitzian, each with Lipschitz constant 2/ $\varepsilon$. Choose $x$ in $C$. It suffices to show that $2 / \varepsilon$ is a Lipschitz constant for $f_{x}$ restricted to $B_{\varepsilon / 2}(0)$, since the positive homogeneity of $f_{x}$ then forces $2 / \varepsilon$ to serve as a global Lipschitz constant. If $2 / \varepsilon$ fails to serve as a local Lipschitz constant at the origin, then there exists $w, y$ in $B_{\varepsilon / 2}(0)$ satisfying

$$
\frac{f_{x}(y)-f_{x}(w)}{\|y-w\|}>2 / \varepsilon \text {. }
$$

Choose $z$ satisfying $y \in w z$ and $\|z-y\|=\varepsilon / 2$. Since $f_{x}$ is convex we obtain

$$
\frac{f_{x}(z)-f_{x}(y)}{\|z-y\|} \geqq \frac{f_{x}(y)-f_{x}(w)}{\|y-w\|}>2 / \varepsilon
$$

so that $f_{x}(z)-f_{x}(y)>1$. Thus $f_{x}(z)>1$. However, $z \in B_{\varepsilon}(0) \subset$ conv $\left(\{x\} \cup B_{\varepsilon}(0)\right)$ requires that $f_{x}(z) \leqq 1$. This contradiction implies that $2 / \varepsilon$ serves as a Lipschitz constant for $f_{x}$.

The proof is now completed by observing that the infimum of a collection of equi-Lipschitzian functions is Lipschitz with the expected Lipschitz constant.

COROLlaRY. Let $C$ be starshaped with respect to the origin. If $\varepsilon$ is positive, then the gauge of $B_{\varepsilon}(C)$ is Lipschitz with Lipschitz constant $2 / \varepsilon$.

Let $m$ denote $n$-dimensional Lebesgue measure. The following structural lemmas will be later used to establish the continuity of Lebesgue measure (relative to the Hausdorff metric) on the family of parallel bodies of a fixed radius in $R^{n}$.

LemMa 2. Let $C$ be a compact starshaped set in $R^{n}$. A point $x$ belongs to bd $B_{r}(C)$ if and only if $\inf \{\|x-y\|: y \in C\}=r$.

Proof. The necessity of the condition is obvious. Conversely, 
suppose that $\inf \{\|x-y\|: y \in C\}=r$. Choosing $q$ arbitrarily in conv $\operatorname{ker} C$, a simple argument involving the convexity of the Euclidean norm yields $R(q, x) \cap B_{r}(C)=q x$. Hence, $x$ must belong to bd $B_{r}(C)$.

The following lemma sharpens an observation of M. Brown [2].

Lemma 3. Let $C$ be a compact set in $R^{n}$. If $r>0$, then

(i) $B_{r}(C)$ is the union of finitely many compact starshaped sets whose convex kernels have nonempty interiors

(ii) $m\{x: \inf \{\|x-y\|: y \in C\}=r\}=0$.

Proof. First, suppose that $r$ exceeds the diameter of $C$. Let $r i$ conv $C$ denote the interior of conv $C$ relative to the smallest flat containing $C$. If $C$ is a single point, $B_{r}(C)$ is starshaped. Otherwise, pick $q$ in $r i \operatorname{conv} C$, and choose $\varepsilon>0$ so small that $B_{\varepsilon}(q) \cap \operatorname{conv} C \subset$ $r i$ conv $C$. We claim that convker $B_{r}(C)$ includes $B_{\varepsilon}(q)$. Choose $x$ in $B_{r}(C)$ and $p$ in $B_{\varepsilon}(q)$ arbitrarily, and select $u$ in $C$ satisfying $\|x-u\| \leqq r$. Since the diameter of $C$ equals the diameter of $\operatorname{conv} C$, we have

$$
\|u-p\| \leqq\|u-q\|+\|q-p\| \leqq \text { diameter of } C<r .
$$

It follows that $\|\lambda x+(1-\lambda) p-u\| \leqq r$ whenever $0 \leqq \lambda \leqq 1$ so that $x p \subset B_{r}(C)$. The proof of (i) is completed in the general case as in [2] by decomposing $C$ into finitely many compact sets $\left\{C_{1}, \cdots, C_{k}\right\}$, each of whose diameters is less than $r$. By Lemma 2 , if $\inf \{\| x-$ $y \|: y \in C\}=r$, then $x$ belongs to bd $B_{r}\left(C_{i}\right)$ for some $i$ in $\{1, \cdots, k\}$. Since $m\left(\operatorname{bd} B_{r}\left(C_{i}\right)\right)=0$ for each $i$ [1], assertion (ii) is established.

\section{Blaschke's theorem via the Arzela-Ascoli theorem.}

THEOREM 1. Let $\left\{C_{k}\right\}$ be a sequence of compact starshaped sets each contained in $\left\{x \in R^{n}:\|x\| \leqq M\right\}$. Then $\left\{C_{k}\right\}$ has a subsequence convergent in the Hausdorff metric to a compact starshaped set.

Proof. If $\left\{p_{k}\right\}$ is a bounded sequence of points in $R^{n}$, then $\left\{C_{k}\right\}$ has a convergent subsequence iff $\left\{C_{k}-p_{k}\right\}$ does. Thus we may assume each $C_{k}$ is starshaped with respect to the origin.

Let $\varepsilon$ be positive and let $f_{k}$ represent the gauge of $B_{\varepsilon}\left(C_{k}\right)$. By the corollary to Lemma 1 , each member of $\left\{f_{k}\right\}$ is Lipschitz with Lipschitz constant 2/ $\varepsilon$. Hence, $\left\{f_{k}\right\}$ is an equicontinuous sequence. Moreover, $\left|f_{k}(w)\right| \leqq\|w\| / \varepsilon$ holds for each $w \in R^{n}$ and $k \in Z^{+}$so that the family of functions is pointwise bounded. By the Arzela-Ascoli theorem $\left\{f_{k}\right\}$ has a subsequence uniformly convergent on compact subsets of $R^{n}$ to a function $f$ which clearly is the guage of the closed 
starshaped set $S=\{w: f(w) \leqq 1\}$. Since $f_{k}(w) \geqq\|w\| /(M+\varepsilon)$, the set $S$ is compact.

Since $f(\varepsilon w /\|w\|) \leqq 1$ for all $w \in R^{n} \backslash\{0\}$, we have $B_{\varepsilon}(0) \subset S$. In addition, the convergence of the subsequence of gauges is uniform on $\{w: f(w)=1\}$. From these observations, it follows that $\{w: f(w)=1\}$ is the Hausdorff limit of a subsequence of $\left\{\left\{\mathrm{w}: f_{k}(w)=1\right\}\right.$, and the corresponding subsequence of $\left\{B_{\varepsilon}\left(C_{k}\right)\right\}$ converges to $S$.

We now produce a convergent subsequence of $\left\{C_{k}\right\}$ itself. First, there exists a subsequence of $\left\{C_{k}\right\}$ whose parallel bodies of radius 1 converge to a compact set $S_{1}$ starshaped with respect to the origin; this subsequence may be filtered to obtain one whose parallel bodies of radius $1 / 2$ converge to $S_{2}$, a subset of $S_{1}$, and so forth. We claim that the subsequence of $\left\{C_{k}\right\}$ whose $i$ th term is the $i$ th term in the $i$ th subsequence as generated above converges to $\bigcap_{i=1}^{\infty} S_{i}$, a compact set starshaped with respect to the origin. Denote this "diagonal" subsequence by $\left\{D_{k}\right\}$.

Let $\varepsilon$ be positive. Choose $m$ so large that $2 / m<\varepsilon$. Since $\left\{B_{1 / m}\left(D_{k}\right): k \in Z^{+}\right\}$converges to $S_{m}$, we have for all but finitely many $k$

$$
B_{\varepsilon}\left(D_{k}\right) \supset B_{2 / m}\left(D_{k}\right)=B_{1 / m}\left(B_{1 / m}\left(D_{k}\right)\right) \supset S_{m} \supset \bigcap_{i=1}^{\infty} S_{i} .
$$

On the other hand, $B_{1 / m}\left(S_{m}\right)$ contains all but finitely many terms of $\left\{D_{k}\right\}$. We claim that if $x \in B_{1 / m}\left(S_{m}\right)$, then $x \in B_{2 / m}\left(\bigcap_{i=1}^{\infty} S_{i}\right)$, a subset of $B_{\varepsilon}\left(\bigcap_{i=1}^{\infty} S_{i}\right)$. If $i>m$, the uniqueness of limits implies $S_{m}=B_{1 / m-1 / i}\left(S_{i}\right)$. Thus, $B_{1 / m}\left(S_{m}\right)=B_{2 / m-1 / i}\left(S_{i}\right)$ for each such $i$. Let $x$ be an arbitrary member of $B_{1 / m}\left(S_{m}\right)$. Choose $y_{i} \in S_{i}$ satisfying $\left\|y_{i}-x\right\| \leqq 2 / m$ for each $i>m$. Since the collection $\left\{S_{i}: i \in Z^{+}\right\}$is nested, $\left\{y_{i}\right\}$ has a subsequence convergent to a point $y$ in $\bigcap_{i=m+1}^{\infty} \operatorname{cl} S_{i}=\bigcap_{i=1}^{\infty} S_{i}$. Since $\|x-y\| \leqq 2 / m<\varepsilon$, we have $x \in B_{\varepsilon}\left(\bigcap_{i=1}^{\infty} S_{i}\right)$. Hence all but finitely many terms of $\left\{D_{k}\right\}$ are contained in $B_{\varepsilon}\left(\bigcap_{i=1}^{\infty} S_{i}\right)$.

4. Lebesgue measure and parallel bodies of a fixed radius. The assignment $C \rightarrow m(C)$ is an upper-semicontinuous function on the metric space of compact subsets of $R^{n}$ under the Hausdorff metric. If $\left\{C_{k}\right\}$ is a sequence of compact sets satisfying $\lim _{k \rightarrow \infty} d\left(C_{k}, C\right)=0$, then the following are equivalent (1) $\lim _{k \rightarrow \infty} m\left(C_{k}\right)=m(C)$ $\lim _{k \rightarrow \infty} m\left(C \Delta C_{k}\right)=0$ (3) $m\left(C \backslash C_{k}\right)=0$ (4) the sequence of characteristic functions corresponding to $\left\{C_{k}\right\}$ converges in measure to the characteristic function of $C$. When these conditions hold, we say that $\left\{C_{k}\right\}$ also converges in measure to $C$. The following results show that Lebesgue measure is continuous on the subspace of parallel bodies of a fixed radius. 
LEMMA 4. Let $\left\{C_{k}\right\}$ be a sequence of compact sets in $R^{n}$ convergent in the Hausdorff metric to a compact set $C$. If $r$ is a fixed positive number, then $\left\{B_{r}\left(C_{k}\right)\right\}$ converges in measure to $B_{r}(C)$.

Proof. Clearly, $\left\{B_{r}\left(C_{k}\right)\right\}$ converges in the Hausdorff metric to $B_{r}(C)$. Hence, we need only show that $\lim _{k \rightarrow \infty} m\left(B_{r}(C) \backslash B_{r}\left(C_{k}\right)\right)=0$.

Suppose that for infinitely many $k$, we have $m\left(B_{r}(C) \backslash B_{r}\left(C_{k}\right)\right)>$ $\delta>0$. By passing to a subsequence, we may assume that this occurs for each $C_{k}$. In each set $B_{r}(C) \backslash B_{r}\left(C_{k}\right)$ pick a compact set $F_{k}$ satisfying $m\left(F_{k}\right)>\delta$. We claim that if $x$ belongs to $\lim \sup \left\{F_{k}\right\}$, then inf $\{\|x-y\|: y \in C\}=r$. Suppose instead that $\|x-y\|<r-\varepsilon$ for some $y$ in $C$ and $\varepsilon>0$. Since $B_{\varepsilon / 2}(x)$ contains points from infinitely many $F_{k}$ and $d\left(C_{k}, C\right) \rightarrow 0$, there exists a positive integer $k$ and points $x_{k}$ in $F_{k}$ and $y_{k}$ in $C_{k}$ satisfying $\left\|x_{k}-x\right\|<\varepsilon / 2$ and $\left\|y_{k}-y\right\|<\varepsilon / 2$ It follows $\left\|x_{k}-y_{k}\right\|<r$, and this contradicts $x_{k} \notin B_{r}\left(C_{k}\right)$. By Lemma $3, m\left(\lim \sup \left\{F_{k}\right\}\right)=0$. We now show that $m\left(\lim \sup \left\{F_{k}\right\}\right) \geqq \delta$; this yields the desired contradiction. Let $\left\{F_{k_{l}}\right\}$ be a convergent subsequence of $\left\{F_{k}\right\}$ with limit $F$. Since $\lim \sup \left\{F_{k}\right\}$ includes $F$, we have

$$
m\left(\lim \sup \left\{F_{k}\right\}\right) \geqq m\left(F^{\prime}\right) \geqq \limsup _{l \rightarrow \infty} m\left(F_{k_{l}}\right) \geqq \delta .
$$

THEOREM 2. Let $\left\{C_{k}\right\}$ be a collection of parallel bodies of a fixed radius convergent in the Hausdorff metric to a compact set $C$. Then $\left\{C_{k}\right\}$ converges in measure to $C$.

Proof. It suffices to show that every subsequence of $\left\{m\left(C \backslash C_{k}\right)\right\}$ has in turn a subsequence convergent to zero. Suppose that $C_{k}=$ $B_{r}\left(E_{k}\right)$ for $k=1,2, \cdots$. Given a subsequence of $\left\{C_{k}\right\}$, choose a subsequence of this subsequence for which the corresponding subsequence of $\left\{E_{k}\right\}$ is convergent. Clearly, the $r$-parallel body of the limit of the above subsequence of $\left\{E_{k}\right\}$ is $C$. An application of Lemma 4 now yields the desired result.

We present a simple application of this result. If $C$ is a compact subset of $R^{n}$, define the visibility function $v_{C}: R^{n} \rightarrow R$ by $v_{C}(x)=$ $m\{y: x y \subset C\}$. For each compact set $C$ of positive measure define $I(C)$ by $\int\left(v_{C} / m(C)^{2}\right) d m$. This set function measures the relative convexity of $C$. Otherwise, set $I(C)=1$. If $\left\{C_{k}\right\}$ is a sequence of compact sets convergent both in the Hausdorff metric and in measure to a compact set $C$, then $I(C) \geqq \lim \sup _{k \rightarrow \infty} I\left(C_{k}\right)$ ) [1]. Theorem 2 now implies that $I$ is upper-semicontinuous on the metric space of parallel bodies of a fixed radius relative to the Hausdorff metric. 


\section{REFERENCES}

1. G. A. Beer, The index of convexity and the visibility function, Pacific J. Math., 44 (1973), 59-67.

2. M. Brown, Sets of constant distance from a planar set, Michigan Math. J., 19 (1972), 321-23.

3. E. Heil, Über dis Auswahlsatze von Blaschke und Arzela-Ascoli, Math.-Phys. Semester., 14 (1967), 169 75.

4. E. Miçhael, Topologies on spaces of subsets, Trans. Amer. Math. Soc., 72 (1951), $152-82$.

5. R. T. Rockafellar, Convex Analysis, Princeton University Press, Princeton, 1970.

6. F. A. Valentine, Convex Sets, McGraw-Hill, New York, 1964.

Received January 3, 1975.

California State University, Los Angeles 



\section{PACIFIC JOURNAL OF MATHEMATICS}

\section{EDITORS}

RICHARD ARENS (Managing Editor) University of California

Los Angeles, California 90024

\section{R. A. BEAUMONT}

University of Washington Seattle, Washington 98105
J. DugundjI Department of Mathematics University of Southern Californı Los Angeles, California 90007

D. Gilbarg AND J. Milgram Stanford University Stanford, California 94305

\section{ASSOCIATE EDITORS}

E. F. BECKENBACH

B. H. NeUMANN

F. WOLF

K. YosHidA

\section{SUPPORTING INSTITUTIONS}

UNIVERSITY OF BRITISH COLUMBIA CALIFORNIA INSTITUTE OF TECHNOLOGY UNIVERSITY OF CALIFORNIA MONTANA STATE UNIVERSITY UNIVERSITY OF NEVADA NEW MEXICO STATE UNIVERSITY OREGON STATE UNIVERSITY UNIVERSITY OF OREGON OSAKA UNIVERSITY

\author{
UNIVERSITY OF SOUTHERN CALIFORNIA \\ STANFORD UNIVERSITY \\ UNIVERSITY OF TOKYO \\ UNIVERSITY OF UTAH \\ WASHINGTON STATE UNIVERSITY \\ UNIVERSITY OF WASHINGTON \\ AMERICAN MATHEMATICAL SOCIETY \\ NAVAL WEAPONS CENTER
}




\section{Pacific Journal of Mathematics}

\section{Vol. 61, No. $1 \quad$ November, 1975}

Jiří Adámek, V. Koubek and Věra Trnková, Sums of Boolean spaces represent every

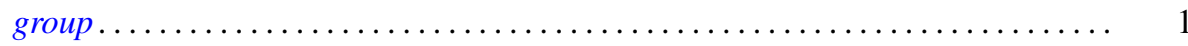

Richard Neal Ball, Full convex l-subgroups and the existence of $a^{*}$-closures of

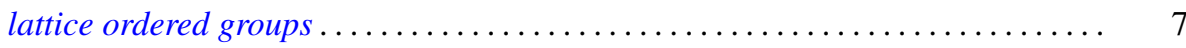

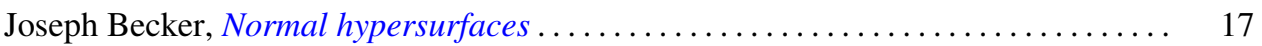

Gerald A. Beer, Starshaped sets and the Hausdorff metric . . . . . . . . . . . . . 21

Dennis Dale Berkey and Alan Cecil Lazer, Linear differential systems with

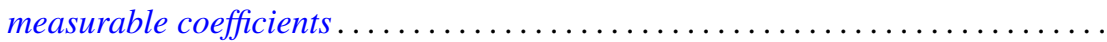

Harald Boehme, Glättungen von Abbildungen 3-dimensionaler

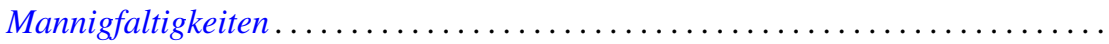

Stephen LaVern Campbell, Linear operators for which $T^{*} T$ and $T+T^{*}$

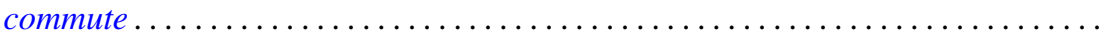

H. P. Dikshit and Arun Kumar, Absolute summability of Fourier series with

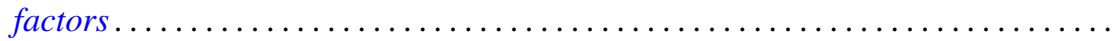

Andrew George Earnest and John Sollion Hsia, Spinor norms of local integral

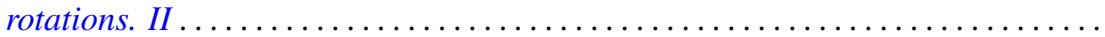

Erik Maurice Ellentuck, Semigroups, Horn sentences and isolic structures .........

Ingrid Fotino, Generalized convolution ring of arithmetic functions . . . . . . . . . . .

Michael Randy Gabel, Lower bounds on the stable range of polynomial rings .......

Fergus John Gaines, Kato-Taussky-Wielandt commutator relations and

characteristic curves

Theodore William Gamelin, The polynomial hulls of certain subsets of $C^{2}$

R. J. Gazik and Darrell Conley Kent, Coarse uniform convergence spaces. . .

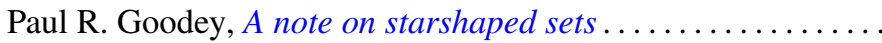

Eloise A. Hamann, On power-invariance

M. Jayachandran and M. Rajagopalan, Scattered compactification for $N \cup\{P\}$. . .

V. Karunakaran, Certain classes of regular univalent functions .

John Cronan Kieffer, A ratio limit theorem for a strongly subadditive set function in a locally compact amenable group .................

Siu Kwong Lo and Harald G. Niederreiter, Banach-Buck measure, density, and uniform distribution in rings of algebraic integers ........

Harold W. Martin, Contractibility of topological spaces onto metric spaces ....

Harold W. Martin, Local connectedness in developable spaces .

A. Meir and John W. Moon, Relations between packing and covering numbers of a tree.

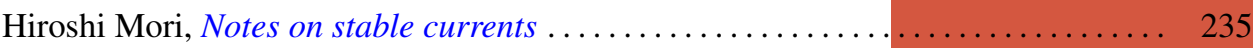

Donald J. Newman and I. J. Schoenberg, Splines and the logarithmic function . . . . 241

M. Ann Piech, Locality of the number of particles operator....

Fred Richman, The constructive theory of $K T$-modules .......

Gerard Sierksma, Carathéodory and Helly-numbers of

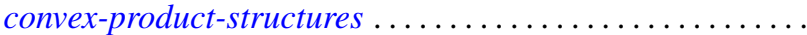

Raymond Earl Smithson, Subcontinuity for multifunctions .... . . 